\title{
PROCESSAMENTO DE FRUTOS DE ABACAXIZEIRO CV SMOOTH CAYENNE: PERFIL DE AÇÚCARES E ÁCIDOS DOS SUCOS E COMPOSIÇÃO NUTRICIONAL DA FARINHA DE CASCAS ${ }^{1}$
}

\author{
SARITA LEONEL 2 , MAGALI LEONEL ${ }^{3}$, ALOÍSIO COSTA SAMPAIO ${ }^{4}$
}

RESUMO-O desenvolvimento de tecnologias que permitam o aproveitamento integral dos frutos é de grande importância para as indústrias. Uma possibilidade de valorização da cultura do abacaxi seria o estímulo ao processamento do fruto para a obtenção do suco associado ao aproveitamento das cascas para a elaboração de farinha, produto de mercado crescente. Um dos fatores de grande importância para a aceitação do suco é o sabor, que se encontra diretamente relacionado ao perfil de ácidos e açúcares presentes. Nesta linha, este trabalho visou a processar frutos de abacaxi cv Smooth Cayenne colhidos em dois estádios de maturação para a obtenção e a caracterização do suco e da farinha de cascas. Os sucos obtidos foram analisados em HPLC para o perfil de açúcares e ácidos orgânicos. As farinhas de casca foram analisadas para rendimento, umidade, cinzas, fibras solúvel e insolúvel, lipídeos, proteína e carboidratos totais. Os resultados obtidos mostraram que frutos em estádio mais avançado de maturação produzem sucos com maior teor de açúcares e menor teor de ácidos, sendo o principal ácido o cítrico, e os açúcares frutose, glicose e sacarose. As farinhas de abacaxi apresentam-se como fontes de fibras insolúveis.

Termos para indexação: Ananas comosus, abacaxi, maturação, resíduo, agregação de valor.

\section{PROCESSING OF PINEAPPLE FRUITS CV SMOOTH CAYENNE: PROFILE OF JUICE SUGARS AND ACIDS AND FLOUR PEEL NUTRITIONAL COMPOSITION}

ABSTRACT- The development of technologies that enable the full use of the fruits is of great importance for the industry. One possible enhancement of the pineapple orchards would be the stimulus for fruit processing to obtain the juice, along with the use of peels for the preparation of flour, which is a product of growing market. One of the major factors for the acceptance of the juice is the flavor, which is directly related to the profile of acids and sugars. In this line, this study aimed to process 'Smooth Cayenne' pineapple fruits, harvested at two maturities stages, for obtaining and characterization of juice and peel flour. The juices were analyzed for the profile in HPLC of sugars and organic acids. The peel flour was analyzed for yield, moisture, ash, soluble and insoluble fiber, lipids, protein and total carbohydrates. The results showed that fruits in the most advanced stage of maturation produce juices with higher sugar content and lower acid content. The main acid was the citric acid and sugars were fructose, glucose and sucrose. The peels flour of pineapple can be used as sources of insoluble fiber.

Index terms: Ananas comosus, pineapple, maturation, residue, value aggregation.

${ }^{1}$ (Trabalho 274-13). Recebido em: 07-08-2013. Aceito para publicação em: 21-01-2014.

${ }^{2}$ Prof. Adjunto, Departamento de Horticultura, FCA/UNESP, Botucatu-SP. E-mail: sarinel@fca.unesp.br

${ }^{3}$ Pesquisadora Doutora, Centro de Raízes e Amidos Tropicais, Universidade Estadual Paulista Júlio de Mesquita Filho", Rua José Barbosa de Barros, 1780, Botucatu-SP, Brasil. CEP: 18610-307. E-mail: mleonel@cerat.unesp.br)

${ }^{4}$ Prof. Adjunto, Departamento de Ciências Biológicas, FC/UNESP, Bauru-SP (e-mail: aloisio@fc.unesp.br) 


\section{INTRODUÇÃO}

O abacaxizeiro (Ananas comosus L. Merril) pertence ao gênero Ananas, que é o mais importante da família Bromeliaceae, do ponto de vista econômico, pois nele estão incluídos os abacaxis. O cultivo de abacaxi concentra-se em seis países que respondem por mais de 59\% da produção mundial. O principal produtor mundial de abacaxi é a Tailândia, seguido pelo Brasil, Filipinas, China, Índia e Costa Rica (THÉ et al., 2010). A cultivar Smooth Cayenne é a mais conhecida e cultivada mundialmente, dada sua qualidade e aceitação comercial.

A composição química do fruto depende do estádio de maturação e de fatores agronômicos e ambientais. Na composição química do abacaxi 'Smooth Cayenne', tem-se considerável teor de açúcares, com 4,5\% de sacarose, 2,21\% de frutose, $1,45 \%$ de glicose, $1,63 \%$ de fibras e 0,016 de proteína, $0,8 \%$ de ácido cítrico e $0,38 \%$ de ácido málico, na base fresca (BARTOLOMÉ et al., 1995).

A qualidade final do fruto é influenciada por práticas culturais no cultivo, na colheita e na pós-colheita, dependendo do estádio de maturação, que influencia na vida útil pós-colheita. Colheitas realizadas antes que os frutos atinjam a completa maturação fisiológica prejudicam o processo de amadurecimento, afetando sua qualidade (PEDREIRA, 2008).

A fome e o desperdício de alimentos são dois dos maiores problemas que o Brasil enfrenta, constituindo-se em um dos paradoxos de nosso País. Neste contexto, diversos trabalhos vêm sendo desenvolvidos, visando ao aproveitamento de partes não tradicionalmente comestíveis de vegetais e frutas, para a elaboração de produtos alimentícios, como sucos, doces, geleias e farinhas (GONDIM et al., 2005).

Em 2004, o Instituto Brasileiro de Frutas (IBRAF) estimou em 350 milhões de litros a produção/consumo de sucos e polpas à base de frutas no Brasil. Como consequência, o aumento deste processamento gera cerca de $40 \%$ dos resíduos agroindustriais, compostos de restos de polpa, casca, caroços ou sementes . O beneficiamento do abacaxi resulta em quantidades consideráveis de resíduos. Aproximadamente 65 a $75 \%$ do fruto são desperdiçados durante sua industrialização (LOUSADA JÚNIOR et al., 2006). Várias pesquisas têm sido desenvolvidas para o aproveitamento dos resíduos do abacaxi (IMANDI et al., 2008; KETNAWA et al., 2010; KETNAWA et al., 2012). Tanaka et al. (1999) empregaram os resíduos como substrato de baixo custo para produção de etanol por fermentação, Imandi et al. (2008) utilizaram enzimas para a extração do suco a partir dos resíduos e Borges et al. (2004) utilizaram os resíduos da indústria de conserva para a obtenção de sucos. Já Ketnawa et al. (2012) utilizaram os resíduos como fonte para a extração de bromelina.

Dentre as opções tecnológicas, destaca-se a secagem de resíduos para obtenção de farinha, como ingrediente alimentar rico em fibras, para incorporação nos mais diversos alimentos, em substituição parcial à farinha de trigo (MATIAS et al., 2005; ABUD; NARAIN, 2009).

Neste contexto, este trabalho teve por objetivo avaliar o perfil de ácidos e açúcares de sucos de abacaxi , bem como produzir e caracterizar uma farinha de casca, com os frutos em dois estádios de maturação, visando a suprir informações para a cadeia produtiva, com a finalidade de ampliação do processamento, associado à valorização do resíduo.

\section{MATERIAL E MÉTODOS}

Frutos de abacaxi cv Smooth Cayenne cultivados em Bauru-SP, foram colhidos em dezembro de 2011, em dois diferentes estádios de maturação, comumente comercializados pelos produtores: subgrupo verde ou verdoso, fruto com casca completamente verde; sub-grupo pintado, fruto com o centro dos frutilhos amarelos.

A área amostrada sofreu processo de diferenciação floral natural em junho/julho de 2011, de modo que os frutos amostrados apresentavam aproximadamente 185 a 210 dias após a indução floral.

Foram processados 30 frutos em cada estádio de maturação, sendo cada fruto considerado uma repetição. Os frutos foram lavados em água clorada e realizou-se o descascamento manual. A polpa dos frutos foi desintegrada em liquidificador industrial e procedeu-se à filtração a vácuo para a separação das fibras e a obtenção do suco. Os sucos foram analisados para o perfil de ácidos orgânicos em cromatógrafo líquido (HPLC), marca Várian, modelo Pró-Star 410, com detector de índice de refração e injetor automático. Foi utilizada a coluna HPX 87H, marca Bio-RAD, fase estacionária resina iônica de hidrogênio (9 micras), fase móvel ácido sulfúrico $0,005 \mathrm{M}$, fluxo de $0,6 \mathrm{~mL} / \mathrm{minuto}$, temperatura de $60^{\circ} \mathrm{C}$. A concentração de ácidos foi determinada através de curvas-padrão.

Para a análise do perfil de açúcares, utilizouse do cromatógrafo líquido (HPLC), marca Várian, modelo Pró-Star 410, com detector de índice de refração e injetor automático, sendo utilizada a 
coluna HPX 87P, marca Bio-RAD, fase estacionária chumbo, 7,8mm, fase móvel água e temperatura da coluna de $80^{\circ} \mathrm{C}$. A concentração dos açúcares foi determinada a partir de curvas-padrão.

No preparo das farinhas, foram utilizados 30 frutos de cada estádio de maturação aparente. Os frutos foram pesados, e realizou-se o descascamento manual. Para fins de balanço de massa, foram pesadas as cascas, polpas e as farinhas originadas de cada fruto, nos dois estádios de maturação, e determinadas as umidades iniciais das cascas e das farinhas.

As cascas foram picadas e desidratadas em estufa de circulação de ar $\left(45^{\circ} \mathrm{C}\right)$ por 48 horas e, decorrido este tempo, efetuou-se a moagem em moinho de facas.

As farinhas obtidas foram caracterizadas quanto aos teores de: umidade, lipídeos, minerais, proteína, carboidratos totais (INSTITUTO ADOLFO LUTZ, 2008) e fibras solúvel e insolúvel pelos métodos AOAC 985.29 e AOAC 991.42 (AOAC, 1997; 2006), sendo realizadas 4 repetições por amostra.

\section{RESULTADOS E DISCUSSÃO}

O sabor do abacaxi depende, entre outros fatores, do conteúdo de açúcares e de ácidos orgânicos e do grau de maturação. A análise do perfil de ácidos orgânicos dos frutos de abacaxi, nos diferentes estádios de maturação, mostrou a presença de ácido málico $\left(0,26\right.$ e $\left.0,33 \mathrm{~g} .100 \mathrm{~mL}^{-1}\right)$, ácido ascórbico $\left(23,5\right.$ e $\left.28,7 \mathrm{mg} .100 \mathrm{~mL}^{-1}\right)$ e ácido cítrico $\left(0,56\right.$ e $\left.0,36 \mathrm{~g} .100 \mathrm{~mL}^{-1}\right)$ para frutos nos estádios verdoso e pintado, respectivamente (Figura 1). Os teores de acidez aumentam desde o início da maturação, atingindo valor máximo em torno de dez dias do período de amadurecimento e, logo após, decrescem acentuadamente (PEDREIRA et al., 2008).

Os ácidos e seus teores relatados na literatura são próximos aos obtidos neste trabalho. Imandi et al. (2008) relatam que a composição ácida do abacaxi é constituída por $0,32 \%$ a $1,22 \%$ de ácido cítrico, $0,1 \%$ a $0,47 \%$ de ácido málico e $0,005 \%$ de ácido oxálico. Câmara et al. (1995), analisando a composição química de sucos e néctares comerciais de abacaxi, observaram a presença de ácido cítrico $\left(0,46\right.$ a $\left.0,96 \mathrm{~g} .100 \mathrm{~mL}^{-1}\right)$, ácido málico $(0,167$ a $\left.0,461 \mathrm{~g} .100 \mathrm{~mL}^{-1}\right)$ e vitamina $\mathrm{C}(14,2$ a $84,2 \mathrm{mg}$ ácido ascórbico.100 $\mathrm{mL}^{-1}$ ). Já Imandi et al. (2008) avaliaram o conteúdo de ácido ascórbico em abacaxi e obtiveram valores entre 10 e $22 \mathrm{mg} .100 \mathrm{~g}^{-1}$.

A análise do perfil de açúcares dos frutos evidenciou a presença de frutose $(2,44 \mathrm{e}$
3,71g.100mL $\left.\mathrm{mL}^{-1}\right)$, glicose $\left(2,95\right.$ e 2,26 g. $\left.100 \mathrm{~mL}^{-1}\right)$ e sacarose $\left(3,24\right.$ e $\left.4,3 \mathrm{~g} .100 \mathrm{~mL}^{-1}\right)$ nos dois estádios de maturação (verdoso e pintado), sendo que os frutos pintados apresentaram maiores teores de açúcares totais (Figura 2). Os valores observados nos frutos analisados foram próximos aos relatados por Bartolomé et al. (1996) para frutos frescos de abacaxi cv. Smooth Cayenne, com 4,5\% de sacarose, $1,45 \%$ de glicose e $2,21 \%$ de frutose, e inferiores aos encontrados por Câmara et al. (1995) para os sucos comerciais de abacaxi, que variaram de 2,19 a $3,19 \mathrm{~g} .100 \mathrm{~mL}^{-1}$ de frutose, 2,21 a $3,31 \mathrm{~g} .100 \mathrm{~mL}^{-1}$ de glicose e 4,13 a 5,51g. $100 \mathrm{~mL}^{-1}$ de sacarose, e os relatados por Thé et al. (2010), que verificaram em frutos de abacaxi Smooth Cayenne teores médios de açúcares de 5,34\% de sacarose e de 3,23\% de glicose. Estas diferenças podem ser devidas às condições climáticas regionais e à época de colheita, que refletem diretamente no estádio de maturação dos frutos.

A análise do balanço de massa para o processo de obtenção das farinhas de abacaxi mostrou que os frutos tiveram peso total de $2,1 \mathrm{~kg}$ a $2,3 \mathrm{~kg}$, e que cerca de $37 \%$ do peso do fruto corresponde às cascas frescas, que apresentam 82,23\% de umidade no estádio menos avançado de maturação (Tabela 1). Thé et al. (2010) observaram variação de 1,8 a $2,1 \mathrm{~kg}$ para frutos de abacaxi cv. Smooth Cayenne colhidos no estádio 2 de maturação, com a região basal do fruto amarela, porém sem ultrapassar duas fileiras. Gondim et al. (2005), analisando cascas de diversas frutas, observaram 78,13\% de umidade para a casca de abacaxi, valor próximo aos encontrados neste trabalho.

A tabela adotada no Brasil, pelo Centro de Qualidade em Horticultura (CEAGESP/SP), para a classificação do abacaxi por peso, separa-o em seis categorias, partindo do mais leve (classe 1), com peso maior ou igual a $0,9 \mathrm{~kg}$ até $1,2 \mathrm{~kg}$, aos mais pesados, com peso superior a $2,4 \mathrm{~kg}$ (classe 6). Segundo esta classificação, os frutos desta pesquisa enquadram-se dentro das categorias 4, com peso entre 1,8 e 2,1 kg, e 5 , entre $2,1 \mathrm{~kg}$ e $2,4 \mathrm{~kg}$.

Ocorreram diferenças significativas para o estádio de maturação quanto ao teor de umidade e rendimento em farinha. Obteve-se maior rendimento em farinha no processamento do abacaxi pintado.

A análise da composição em nutrientes das farinhas de casca evidenciou diferenças significativas para os teores de lipídeos, carboidratos totais e fibra insolúvel, entre os estádios de maturação aparente (Tabela 2).

Para o teor de matéria graxa, um dos principais componentes em produtos alimentícios, 
os resultados mostraram aumento deste componente com o avanço no estádio de maturação do fruto. Gondim et al. (2005) relatam 0,55\% de lipídeos para a casca fresca de abacaxi, valor próximo ao encontrado para o estádio verdoso, quando considerado na base úmida $(0,38 \%)$.

A análise das farinhas mostrou serem estas excelentes fontes de fibras nutricionais, essencialmente insolúveis. As fibras alimentares têm cada qual efeito fisiológico diferente. Em geral, as fibras solúveis em água (pectinas, gomas, mucilagens e certas hemiceluloses) retardam a passagem intestinal, o esvaziamento gástrico e a absorção de glicose, ajudando a reduzir o colesterol no soro sanguíneo. As fibras insolúveis em água (lignina, celulose e algumas hemiceluloses) aceleram o trânsito intestinal, aumentam o peso das fezes, desaceleram a hidrólise do amido e retardam a absorção da glicose, contribuindo para a redução do risco de alguns males do cólon (LEONEL et al., 1999).

Mattos e Martins (2000), citando a quantidade de fibras em diferentes alimentos, adotaram a seguinte classificação: alimentos com teor muito alto de fibras (mínimo 7g fibras $100 \mathrm{~g}^{-1}$ ); alto (4,5 a 6,9g fibras $\left.100 \mathrm{~g}^{-1}\right)$; moderado (2,4 a 4,4g fibras 100 $\mathrm{g}^{-1}$ ) e baixo (inferior a 2,4g fibras $100 \mathrm{~g}^{-1}$ ). Portanto, a farinha de cascas de abacaxi é um produto de teor muito alto de fibras, sendo que a ingestão de 20 $\mathrm{g}$ de farinha forneceria de 7,51g a $8,17 \mathrm{~g}$ de fibras alimentares, o que corresponde a $24,4 \%$ a $26,55 \%$ do valor diário de referência $(30 \mathrm{~g})$ com base em uma dieta de 2.500 calorias.

Quanto aos teores de proteína, os teores encontrados foram próximos ao teor de 1,45\% relatado para a casca de abacaxi, com 78,13\% de umidade (GONDIM et al., 2005).

A ingestão diária recomendada (IDR) é a quantidade de proteína, vitaminas e minerais que deve ser consumida diariamente para atender às necessidades nutricionais da maior parte dos indivíduos e grupos de pessoas de uma população sadia. Considerando a ingestão diária recomendada para adultos de $50 \mathrm{~g}$. dia ${ }^{-1}$ de proteína, o consumo de $20 \mathrm{~g}$. dia $^{-1}$ de farinha de casca de abacaxi representaria de $1,66 \%$ a $1,78 \%$ da IDR.

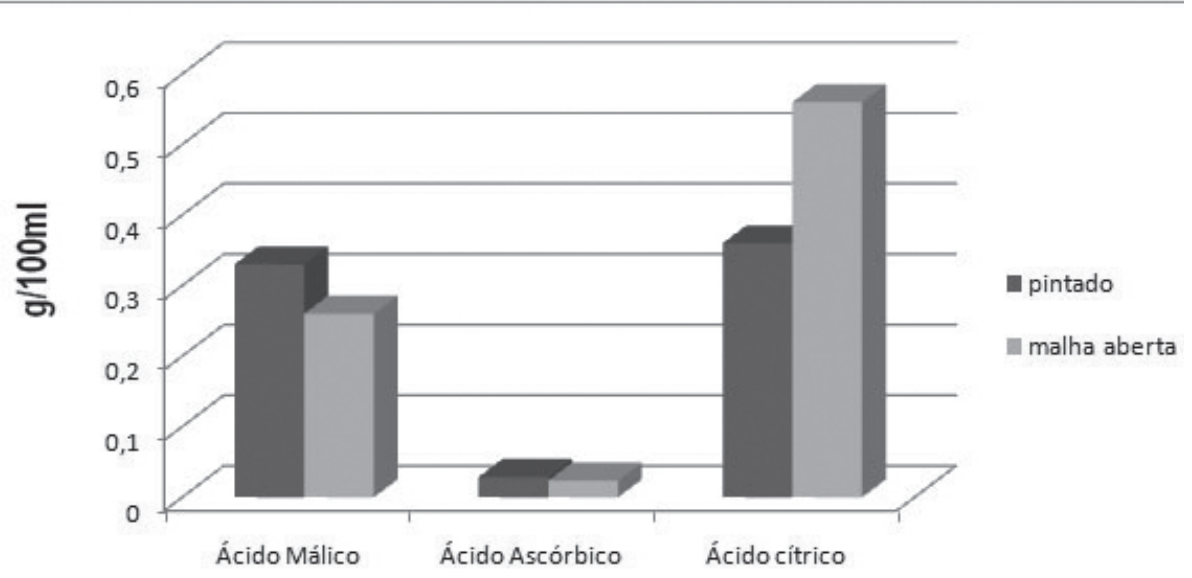

FIGURA 1- Perfil de ácidos orgânicos de frutos de abacaxi cv Smooth Cayenne em dois estádios de maturação (30 amostras/estádio). 


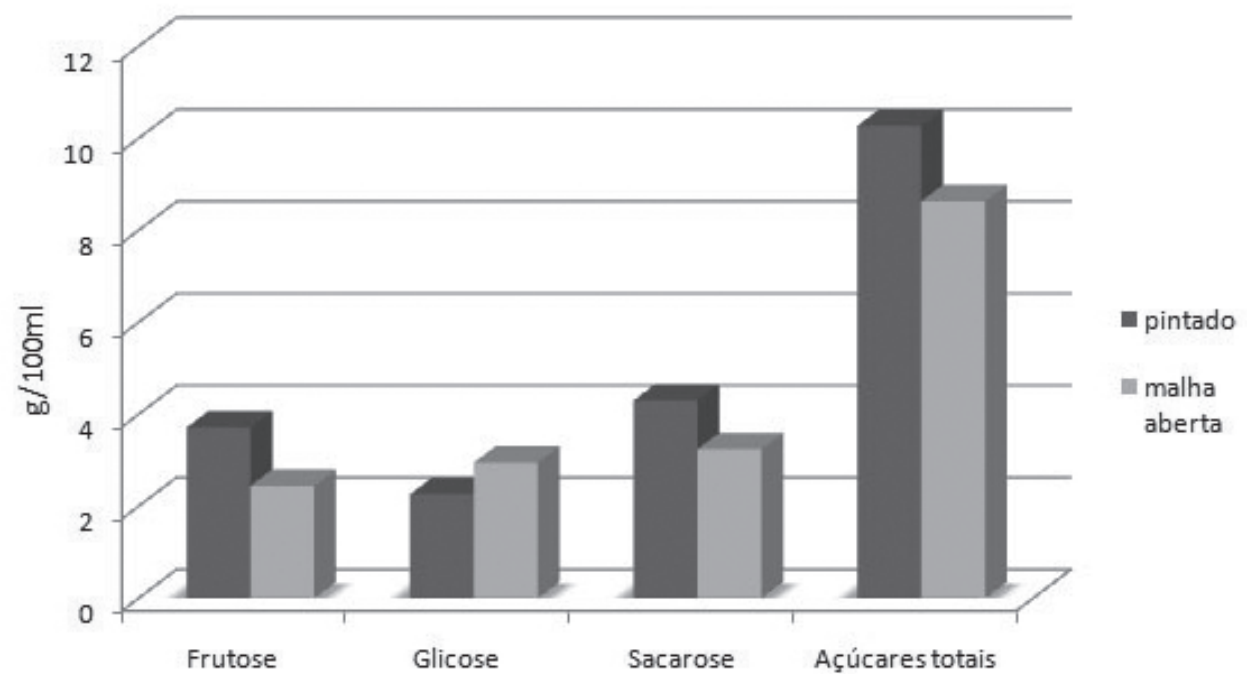

FIGURA 2- Perfil de açúcares de frutos de abacaxi cv Smooth Cayenne em dois estádios de maturação (30 amostras/estádio).

TABELA 1- Resultados médios das análises do balanço de massa e abacaxi cv Smooth Cayenne(30 frutos/ estádio).

\begin{tabular}{lcccc}
\hline & Malha aberta & Pintado & CV (\%) & DMS 5\% \\
\hline Peso casca (g) & $786,59 \mathrm{a}$ & $693,09 \mathrm{a}$ & 15,7 & 109,15 \\
Peso polpa (g) & $1.031,69 \mathrm{a}$ & $1.221,59 \mathrm{a}$ & 15,81 & 188,29 \\
Peso total com coroa $(\mathrm{g})$ & $2.292,3 \mathrm{a}$ & $2.105,68 \mathrm{a}$ & 14,7 & 277,3 \\
\% casca & $37,56 \mathrm{a}$ & $36,18 \mathrm{a}$ & 7,92 & 2,74 \\
Umidade casca (\%) & $82,23 \mathrm{a}$ & $78,46 \mathrm{~b}$ & 2,03 & 1,53 \\
Peso farinha (g) & $122,01 \mathrm{a}$ & $132,23 \mathrm{a}$ & 14,86 & 17,74 \\
Rendimento de farinha (\%) & $15,51 \mathrm{~b}$ & $19,07 \mathrm{a}$ & 8,28 & 1,35 \\
\hline
\end{tabular}

*Médias seguidas de mesma letra na linha não diferem entre si, no nível de 5\%, pelo teste de Tukey.

TABELA 2- Composição nutricional médias das farinhas de abacaxi (30 frutos/estádio).

\begin{tabular}{ccccc}
\hline g/100g & Malha aberta & Pintado & CV (\%) & DMS 5\% \\
\hline Umidade & $11,85 \mathrm{a}$ & $11,48 \mathrm{a}$ & 3,05 & 0,81 \\
Cinzas & $4,80 \mathrm{a}$ & $4,83 \mathrm{a}$ & 3,85 & 0,42 \\
Lipídeos & $1,87 \mathrm{~b}$ & $4,64 \mathrm{a}$ & 8,3 & 0,61 \\
Proteína & $4,45 \mathrm{a}$ & $4,15 \mathrm{a}$ & 7,8 & 0,76 \\
Carboidratos totais & $43,36 \mathrm{~b}$ & $47,34 \mathrm{a}$ & 3,66 & 3,76 \\
Fibra solúvel & $2,49 \mathrm{a}$ & $2,31 \mathrm{a}$ & 1,72 & 0,93 \\
Fibra insolúvel & $38,34 \mathrm{a}$ & $35,26 \mathrm{~b}$ & 1,36 & 1,14 \\
\hline
\end{tabular}

*Médias seguidas de mesma letra na linha não diferem entre si, no nível de 5\%, pelo teste de Tukey. 


\section{CONCLUSÕES}

Os resultados obtidos permitiram concluir que: o ácido cítrico é o principal ácido de frutos de abacaxi cv Smooth Cayenne, seguido pelos ácidos málico e ascórbico. Frutos em estádio mais avançado de maturação apresentam maior teor de açúcares e menor teor de ácidos, sendo os principais açúcares a frutose, a glicose e a sacarose. As farinhas de abacaxi apresentam-se como fontes de fibras insolúveis, sendo que as farinhas obtidas de frutos em menor estádio de maturação apresentam menor teor de lipídeos e carboidratos totais.

\section{REFERENCIAS}

ABUD, A.K.S., NARAIN, N. Incorporação de farinha de resíduo do processamento de polpa de fruta em biscoitos: uma alternativa de combate ao desperdício. Brazilian Journal of Food Technology, Campinas, v.12, n.4, p.257-265, 2009.

ASSOCIATION OF OFFICIAL ANALYTICAL CHEMISTRY-AOAC. Official methos of analysis of Association of Official Analytical Chemistry. 16 th ed. Arlington, 1997. Volume 1.

ASSOCIATION OF OFFICIAL ANALYTICAL CHEMISTRY-AOAC. Official methos of analysis of Association of Official Analytical Chemistry. 18 th ed. Arlington, 2006. 1141p.

BARTOLOMÉ, A.P.; RÚPEREZ, P.; FÚSTER, C. Changes in soluble sugars of two pineapple fruit cultivars during frozen storage. Food Chemistry, Barking, v.56, n.2, p.163-166, 1996.

BARTOLOMÉ, A. P.; RUPÉREZ, P.; FÚSTER, C. Pineapple fruit: morphological characteristics, chemical composition and sensory analysis of red Spanish and Smooth cayenne cultivars. Food Chemistry, Barking, v. 53, p. 75-79, 1995.

BORGES, C.D.; CHIM, J.F.; LEITÃO, A.M.; PEREIRA, E.; LUVIELMO,.M. Produção de suco de abacaxi obtido a partir dos resíduos da indústria conserveira. Boletim do Centro de Pesquisa de Processamento de Alimentos, Curitiba, v.22, n.1, p.25-34, 2004.

CÂMARA, M.; DIEZ, C.; TORIJA, E. Chemical characterization of pineapple juices and nectars. Principal components analysis. Food Chemistry, Barking, v.54, p.93-100, 1995.
GONDIM, J.A.M.; MOURA, M.F.V.; DANTAS, A.S.; MEDEIROS, R.L.S.; SANTOS, K.M. Composição centesimal e de minerais em cascas de frutas. Ciência e Tecnologia de Alimentos, Campinas, v.25, n.4, p.825-827, 2005.

IMANDI, S.B.; BANDARU, V.V.; SOMALANKA, S.R.; BANDARU, S.R.; GARAPATI, H.R. Application of statistical experimental designs for the optimization of medium constituents for the production of citric acid from pineapple waste. Bioresource Technology, Essex, v.99, p.4.4454.450, 2008.

INSTITUTO ADOLFO LUTZ. Normas analíticas do Instituto Adolfo Lutz: métodos químicos e físicos para análises de alimentos. São Paulo, 2008.

KETNAWA, S.; CHAIWUT, P.; RAWDKUEN, S. Pineapple wastes: a potential source for bromein extraction. Food and Bioproducts Processing, Rugby v.90, p.385-391, 2012.

KETNAWA, S.; RAWDKUEN, S.; CHAIWUT, P. Two phase partitioning and collagen hydrolysis of bromelain from pineapple peel Nang Lae cultivar. Biochemical Engineering. Journal, Amsterdan, v.52, p.205-211, 2010.

LEONEL, M.; CEREDA, M. P; ROAU, X. Aproveitamento do resíduo da produção de etanol a partir de farelo de mandioca, como fonte de fibras dietéticas. Ciência e Tecnologia de Alimentos, Campinas, v.19, n.2, p.241-245, 1999.

LOUSADA JÚNIOR, J. E.; COSTA, J. M. C.; NEIVA, J. N. M.; RODRIGUEZ, N. M. R. Caracterização físicoquímica de subprodutos obtidos do processamento de frutas tropicais visando a seu aproveitamento na alimentação animal. Revista Ciência Agronômica, Fortaleza, v.37, n.1, p.70-76, 2006.

MATIAS, M. F. O.; OLIVEIRA, E. L.; MARGALHÃES, M. M. A.; GERTRUDES, E. Use of fibers obtained from the cashew (Anacardium ocidentale, L) and guava (Psidium guayava) fruits for enrichment of food products. Brazilian Archives of Biology and Technology, Curitiba, v. 48, p. 143-150, 2005. Número especial

MATTOS, L.L.; MARTINS, I.S. Dietary fiber consumption in an adult population. Revista Saúde Pública, São Paulo, v.34, n.1, p.50-55, 2000. 
PEDREIRA, A.C.C.; NAVES, R.V.; NASCIMENTO, J.L. Variação sazonal da qualidade do abacaxi cv. Pérola em Goiânia, Estado de Goiás. Pesquisa Agropecuária Tropical, Goiânia, v. 38, n. 4, p. 262-268, 2008

TANAKA, K.; HILARY, Z.D.; ISHIZAKI, A. Investigation of utility of pienaple juice and pineapple waste material as low-cost substrate for ethanol fermentation by Zynmomonas mobilis. Journal of Bioscience and Bioengineering, Osaka, v.87, p.642-646, 1999.
THÉ, P.M.P.; NUNES, R.P.; MOREIRA DA SILVA, L.I.M.; ARAÚJO, B.M. Características físicas, físico-químicas, químicas e atividade enzimática de abacaxi Cv Smoth Cayene recém-colhido. Alimentos e Nutrição, Araraquara, v.21, n.2, p.273-281, 2010. 